Performance of QuantiFERON-TB Gold Plus assays in children and adolescents at risk of tuberculosis: a cross-sectional multi-center study

${ }^{1}$ Aleix SOLER-GARCIA, ${ }^{1}$ Anna GAMELL, ${ }^{3,4}$ Tomàs PÉREZ-PORCUNA, ${ }^{4}$ Antoni SORIANO-ARANDES, ${ }^{5}$ Begoña SANTIAGO, ${ }^{6}$ Teresa TÓRTOLA, ${ }^{7}$ María Jesús RUIZ-SERRANO, ${ }^{8,9}$ José Javier KORTA-MURUA, ${ }^{10}$ Matilde BUSTILLO-ALONSO, ${ }^{11}$ Maria Isabel GARROTE-LLANOS, ${ }^{12}$ Paula RODRÍGUEZ-MOLINO, ${ }^{13}$ Ana Isabel PIQUERAS, ${ }^{14-16}$ Alfredo TAGARRO, ${ }^{17}$ Manuel MONSONÍS, ${ }^{18,19 *}$ Marc TEBRUEGGE, ${ }^{1,16,20,21 *}$ Antoni NOGUERA-JULIAN on behalf of the QFT-Plus Study Group of the Spanish Pediatric TB Research Network (pTBred; see Appendix 1)

(1) Malalties Infeccioses i Resposta Inflamatòria Sistèmica en Pediatria, Unitat d'Infeccions, Servei de Pediatria, Institut de Recerca Pediàtrica Hospital Sant Joan de Déu, Barcelona, Spain.

(2) Atenció Primària, Fundació Assistencial Mútua Terrassa, Terrassa, Spain.

(3) Unitat de Salut Internacional, Departament de Pediatria, Fundació Recerca Mútua Terrassa, Atenció Primària, Hospital Universitari Mútua de Terrassa, Universitat de Barcelona, Terrassa, Spain.

(4) Pediatric Infectious Diseases and Immunodeficiencies Unit, Hospital Universitari Vall d'Hebron, Vall d'Hebron Research Institute, Barcelona, Spain.

(5) Paediatric Infectious Diseases Unit, Gregorio Marañón Hospital, Madrid, Spain.

(6) Unitat de Micobactèries, Laboratori Supranacional de Referència de l'OMS per a la Tuberculosi, Hospital Universitari Vall d'Hebron, Barcelona, Spain.

(7) Clinical Microbiology and Infectious Diseases Department, Hospital General Universitario Gregorio Marañón, Madrid, Spain.

(8) Servicio de Pediatría, Hospital Universitario Donostia-Instituto BioDonostia, San Sebastián, Spain.

(9) Departamento de Pediatría, Facultad de Medicina, EHU-UPV, San Sebastián, Spain.

(10) Pediatrics Department, Hospital Universitario Miguel Servet, Zaragoza, Spain.

(11) Pediatric Infectious Diseases Unit, Department of Pediatrics, Hospital de Basurto, Bilbao, Spain.

(12) Pediatric Infectious and Tropical Diseases Department, Hospital La Paz, Madrid, Spain.

(13) Pediatric Infectious Diseases Unit, Hospital Universitario y Politécnico La Fe, Valencia, Spain.

(14) Servicio de Pediatría, Hospital Universitario Infanta Sofía, San Sebastián de los Reyes, Madrid, Spain.

(15) Fundación para la Investigación Biomédica del Hospital 12 de Octubre, Universidad Europea de Madrid, Madrid, Spain.

(16) Red de Investigación Translacional en Infectología Pediátrica, RITIP, Madrid, Spain.

(17) Servei de Microbiologia, Hospital Sant Joan de Déu, Barcelona, Spain.

(18) Department of Infection, Immunity and Inflammation, UCL Great Ormond Street Institute of Child Health, University College London, London, United Kingdom.

(19) Department of Paediatrics, University of Melbourne, Parkville, Australia.

(20) Departament de Pediatria, Universitat de Barcelona, Barcelona, Spain.

(21) CIBER de Epidemiología y Salud Pública, CIBERESP, Madrid, Spain.

(*) Both authors shared credit for senior authorship. 


\section{Corresponding author:}

\section{Dr. Antoni NOGUERA-JULIAN}

Unitat d'Infeccions, Servei de Pediatria, Hospital Sant Joan de Déu

Passeig Sant Joan de Déu 2, 08950 Esplugues (Spain)

Tel.: +34 932804000 (ext. 80063); fax: +34 932033959

Email: ton@sjdhospitalbarcelona.org

Word count: 3555 words

Keywords: active tuberculosis; child; interferon-gamma release assay; latent tuberculosis; sensitivity; tuberculin skin test

\section{Data availability:}

Data are available upon reasonable request to the corresponding author.

\section{Contributors:}

TPP, ASA, ANJ and MT conceived the original idea and supervised the development of the project. ASG, AG, TPP, ASA, BS, MJRS, JJKM, MBA, MIGL, PRM, AIP, AT and ANJ collaborated in the enrollment of patients and the clinical interpretation of individual results. MM and TT supervised the development of the immunological experiments. ASG, AG and ANJ performed the analysis of the data and designed the figures. ASG and AG wrote the first draft of the manuscript, with support from ANJ and MT. All authors discussed the results and commented on and approved the final version of the manuscript. 


\begin{abstract}
Introduction. The QuantiFERON-TB Gold Plus (QFT-Plus) assay, which features two antigen-stimulated tubes (TB1 and TB2) instead of a single tube used in previous generation interferon-gamma release assays (IGRAs), was launched in 2016. Despite this, data regarding the assay's performance in the paediatric setting remain scarce. This study aimed to determine the performance of QFT-Plus in a large cohort of children and adolescents at risk of tuberculosis (TB) in a low-burden setting.
\end{abstract}

Methods. Cross-sectional, multi-centre study at healthcare institutions participating in the Spanish Pediatric TB Research Network, including patients <18 years who had a QFT-Plus performed between September 2016 and June 2020.

Results. Of 1726 patients (52.8\% male; median age: 8.4 years), 260 (15.1\%) underwent testing during contact tracing, 288 (16.7\%) on clinical/radiological suspicion of TB disease (TBD), 649 (37.6\%) during new-entrant migrant screening and $529(30.6 \%)$ prior to initiation of immunosuppressive treatment. Overall, the sensitivity of QFT-Plus for TBD (n=189) and for latent TB infection (LTBI; $n=195)$ was $83.6 \%$ and $68.2 \%$, respectively. The agreement between QFT-Plus TB1 and TB2 antigen tubes was excellent $(98.9 \%$; $\kappa=0.961)$. Only 5 (2.5\%) patients with TBD had discordance between TB1 and TB2 results (TB1+/TB2-, n=2; TB1-/TB2+, n=3). Indeterminate assay results $(\mathrm{n}=54 ; 3.1 \%)$ were associated with young age, lymphopenia and elevated C-reactive protein concentrations.

Conclusions. Our non-comparative study indicates that QFT-Plus does not have greater sensitivity than previous generation IGRAs in children, in both TBD and LTBI. In TBD the addition of the second antigen tube, TB2, does not enhance the assay's performance substantially.

\title{
What is the key question?
}

Does QuantiFERON-TB Gold Plus perform better than previous interferon-gamma release assays in children and adolescents at risk of tuberculosis in a low-endemic setting?

What is the bottom line?

QuantiFERON-TB Gold Plus performed very similarly to previous interferon-gamma release assays, and the second antigen tube did not significantly increase the assay sensitivity in the diagnosis of active tuberculosis.

\section{Why read on?}

We report the findings of the currently largest multi-center study on the performance of QuantiFERON-TB Gold Plus in 1726 children and adolescents investigated for tuberculosis infection in Spain. 


\section{INTRODUCTION}

Tuberculosis (TB) is the leading cause of death from an infectious disease worldwide.[1,2] It is estimated that 1.1 million children develop tuberculosis disease (TBD) anually.[2,3] In paediatric patients, microbiological confirmation of TBD is difficult to achieve, due to the its paucibacillary nature and difficulties in obtaining sputum in young children.[4,5] Therefore, the diagnosis of TBD in children is often based on a combination of clinical and radiological findings, epidemiologic risk factors and a positive tuberculin skin test (TST) or interferon-gamma release assay (IGRA) result.[6]

IGRAs are in vitro immunoassays based on the detection of interferon-gamma produced by $\mathrm{T}$ cells in response to stimulation with Mycobacterium tuberculosis-specific peptides.[7] In resource-rich countries, IGRAs are widely used for LTBI screening and as adjunctive tools in the diagnostic work-up of suspected TBD.[8] The main advantage of IGRAs compared to TST lies in their greater specificity, with less potential confounding by prior bacillus Calmette-Guérin (BCG) vaccination and non-tuberculous mycobacterial infections.[9]

In Europe, QuantiFERON-TB assays (QFT; Cellestis/Qiagen, Hilden, Germany) are more commonly used than T-SPOT.TB assays (Oxford Immunotec, Abingdon, UK).[8] In 2016, the QuantiFERON-TB Gold Plus (QFTPlus) assay was launched, replacing the previous-generation QuantiFERON-TB Gold in-Tube (QFT-GIT) assay. QFT-Plus assays require an extra millilitre of blood and comprise of 4 tubes (vs. 3 tubes in QFT-GIT): a negative-control (nil) tube, a positive-control (mitogen) tube and two antigen tubes (TB1 and TB2). TB1, intended to elicit CD4+ $\mathrm{T}$ cell responses, contains the original QFT ESAT-6 and CFP-10 antigens but lacks TB7.7, which was included in QFT-GIT. TB2 is a new tube that contains the same two mycobacterial peptides and additional shorter peptides aimed at eliciting CD8+ $\mathrm{T}$ cell responses.[10,11]

In adults, a large meta-analysis estimated the pooled sensitivity of QFT-Plus in the diagnosis of TBD to be 93\%,[12] and head-to-head comparisons between QFT-Plus and QFT-GIT did not identify clinically-relevant differences between the two assays.[13,14] Recent data suggest that QFT-Plus assay performance is not impaired in HIV-infected adults, but patients with immune-mediated inflammatory diseases show decreased interferongamma responses when compared to healthy controls. $[15,16]$ However, currently data on the performance of QFT-Plus in children remain scarce, and no meta-analysis has been performed yet.[17-21]

We recently reported that QFT-Plus is no more sensitive or specific than previous QFT assays in children and adolescents with TBD.[20,22] In this study, we aimed to determine the performance of QFT-Plus in a large cohort of paediatric patients at risk of TB in a low-burden country, by including children undergoing latent TB infection (LTBI) screening as part of contact tracing, migrant screening and screening prior to initiation of immunosuppressive medication.

\section{METHODS}

We performed a cross-sectional study within the Spanish Pediatric TB Research Network (pTBred),[23] which currently includes 83 participating centers. Data were collected using REDCap tools, hosted at Instituto de 
Investigación Sanitaria Gregorio Marañón in Madrid.[24] Approval for pTBred was obtained from the Hospital Carlos III Ethics Committee (Madrid, ref. P13/12). Informed consent was obtained from parents/guardians and assent from adolescents $>11$ years-of-age at inclusion. In Spain, the incidence of TBD has continuously been decreasing over the last 20 years. In 2019, the incidence was 9.3 cases per 100,000 persons in the general population, and 4.2 per 100,000 in children < 15 years-of-age.[25] BCG vaccination has not been part of the routine immunisation program in Spain since 1980, except for the Basque Country, where neonatal BCG vaccination continued until 2013.

For this study, we enrolled patients $<18$ years-of-age who underwent a QFT-Plus assay at a pTBred participating center between September 2016 and June 2020 due to any of the following indications: i) contact tracing (CTR), ii) clinical or radiological suspicion of TBD (CRS), iii) new-entrant migrant screening (NMS) or iv) prior to initiation of immunosuppressive treatment (IIT). Some participating centres recruited all eligible patients consecutively, while others used convenience sampling. Patients with a previous history of TBD or LTBI were excluded from participation.

All QFT-Plus assays were performed in fully-accredited diagnostic laboratories at each participating institution, and their results interpreted according to manufacturer's instructions.[26] In brief, the test result was considered positive when TB1 or TB2 antigen tube minus nil tube interferon-gamma concentrations were $\geq 0.35 \mathrm{IU} / \mathrm{ml}$ and $\geq 25 \%$ of the nil tube concentration; negative when they were $<0.35 \mathrm{IU} / \mathrm{ml}$; and indeterminate when the nil tube concentration was $>8 \mathrm{IU} / \mathrm{ml}$ (i.e. failed negative-control) and/or when the mitogen tube minus nil tube concentration was $<0.5 \mathrm{IU} / \mathrm{ml}$ (i.e. failed positive-control).

TSTs were performed by intradermal injection of 2 tuberculin-units of purified protein derivative (PPD RT23; Statens Serum Institut; Copenhagen, Denmark), with results read after 48-72 hours. The cut-offs for a positive TST result were based on national guidelines: $\geq 10 \mathrm{~mm}$ induration in NMS children and $\geq 5 \mathrm{~mm}$ in all other groups, irrespective of BCG vaccination status.[27]

For the purpose of the study, haemoglobin concentrations and lymphocyte counts were classified as low, normal or high as previously described.[28] In addition, data on C-reactive protein (CRP; normal value <15 mg/l) and erythrocyte sedimentation rate (ESR; normal value $<15 \mathrm{~mm}$ ) at the time when the QFT-Plus was performed were collected if available.

Patients were categorised according to their final diagnosis regarding TB infection, as follows: a) TB-uninfected: patients without signs or symptoms consistent with TBD and with a negative QFT-Plus and a negative TST result (if performed), b) LTBI: patients without signs or symptoms consistent with TBD and a positive QFT-Plus and/or positive TST result, and c) TBD. In pTBred, the diagnosis of TBD is based on epidemiological, clinical, radiological, and microbiological findings according to published consensus criteria,[29] independent of TST and IGRA results. TBD was further classified as i) microbiologically-confirmed or unconfirmed, ii) intrathoracic 
or extrathoracic, and iii) severe or non-severe, based on published consensus definitions.[30] Some of the TBD cases $(n=158)$ included in the present study had been included in previous reports.[20,22]

Quantitative variables are reported as medians and interquartile ranges (IQR), and categorical variables as proportions with $95 \%$ confidence intervals (95\%CI). Missing data were handled with the complete case analysis method. Sensitivity was calculated based on the proportion of positive QFT-Plus results; indeterminate results were considered negative for those calculations. Bivariate analysis was performed using Chi-square and Fisher's exact tests for categorical variables, and Student $t$ and ANOVA tests for quantitative variables. Non-normally distributed variables were compared with Mann-Whitney $U$ and Kruskal-Wallis tests. Spearman rho test was used to analyse relationships between quantitative data. Binary logistic regression modelling was used for multivariate analysis to test the effect of covariates as risk factors for determinate (i.e. positive or negative) versus indeterminate QFT-Plus results. The results are presented as unadjusted odds ratios (OR) and adjusted odds ratios (aOR) with $95 \%$ CIs.

Total percentage agreement and Cohen kappa coefficient $(\kappa)$ with standard error (SE) were used to quantify concordance between QFT-Plus and TST results, and between qualitative TB1 and TB2 results; patients with indeterminate results were excluded from those analyses. Strength of agreement was defined as poor $(\kappa \leq 0.2)$, fair $(0.2<\kappa \leq 0.4)$, moderate $(0.4<\kappa \leq 0.6)$, good $(0.6<\kappa \leq 0.8)$ and excellent $(\kappa>0.8)$. All statistical analyses were performed using SPSS V24 (IBM; Armond, NY), with statistical significance defined as a $p$-value $<0.05$. Graphs were generated with SPSS and Prism 8 (GraphPad; San Diego, CA).

\section{RESULTS}

\section{Characteristics of the study population}

Of an initial study population of 1736 children and adolescents, 10 patients were excluded due to a previous diagnosis of TBD $(n=6)$ or LTBI $(n=4)$. Therefore, the final study cohort comprised 1726 patients [52.8\% male; median (IQR) age: 8.4 (4.3-12.8) years; Figure 1]. The indications for performing QFT-Plus were: CTR $(\mathrm{n}=260$, 15.1\%), CRS $(n=288,16.7 \%)$, NMS $(n=649,37.6 \%)$ and IIT $(n=529,30.6 \%)$. There was no significant difference between those 4 patient groups with regards to gender, patient origin and BCG vaccination status, but the age distribution and family origin differed significantly (Table 1).

The majority of patients were born in Spain $(n=1280,74.2 \%)$, but fewer than one third $(n=521,30.2 \%)$ of the families were native. Among the 410 children born outside Spain, the most frequent region of origin was South America, followed by Northern Africa and Southern Asia (Table 1).

Haemoglobin concentrations, lymphocyte counts, CRP and ESR values were available for 58.8\%, 58.6\%, 47.0\% and $40.5 \%$ of the patients, respectively. Low haemoglobin concentrations were mainly observed in CRS and IIT patients, while the highest proportion of lymphopenia was observed in IIT patients (Supplementary Table 1). 
QFT-Plus test results were negative in 1381 (80.0\%) patients, positive in 291 (16.9\%), and indeterminate in 54 (3.1\%). The final diagnoses were as follows: $1342(77.8 \%)$ patients were categorised as TB-uninfected, 195 (11.3\%) as LTBI, and $189(11.0 \%)$ as TBD. The highest rates of TBD were observed in CTR and CRS children; none of the IIT patients were diagnosed with TBD (Table 1). The highest proportion of patients with LTBI was observed in the CTR group. 
Table 1. Comparison of baseline characteristics of the study participants according to test indication. Data are shown as number (percentage),

except where stated otherwise.

\begin{tabular}{|c|c|c|c|c|c|c|c|}
\hline \multicolumn{2}{|l|}{ Test indication } & Contact tracing & $\begin{array}{c}\text { Clinical / radiological } \\
\text { suspicion of TB }\end{array}$ & $\begin{array}{l}\text { New-entrant } \\
\text { screening }\end{array}$ & $\begin{array}{l}\text { Immunosuppressive } \\
\text { treatment screening }\end{array}$ & Total & $p$-value \\
\hline \multicolumn{2}{|l|}{ n (\%) } & $260(15.1)$ & $288(16.7)$ & $649(37.6)$ & $529(30.6)$ & $1726(100)$ & \\
\hline \multirow[t]{2}{*}{ Age } & $<5$ years & $118(45.4)$ & $107(37.2)$ & $178(27.1)$ & $112(21.2)$ & $515(29.8)$ & \multirow[t]{2}{*}{$<0.001$} \\
\hline & $\geq 5$ years & $142(54.6)$ & $181(62.8)$ & $471(72.6)$ & $417(78.8)$ & $1211(70.2)$ & \\
\hline \multirow[t]{2}{*}{ Sex } & Male & $136(52.3)$ & $157(54.3)$ & $348(53.6)$ & $271(51.2)$ & $911(52.8)$ & \multirow[t]{2}{*}{0.816} \\
\hline & Female & $123(47.5)$ & $132(45.7)$ & $301(46.4)$ & $258(48.8)$ & $815(47.2)$ & \\
\hline \multirow[t]{3}{*}{ BCG status } & Unvaccinated & $189(72.7)$ & $214(74.3)$ & $476(73.4)$ & $397(75.0)$ & $1276(73.9)$ & \multirow[t]{3}{*}{0.567} \\
\hline & Vaccinated & $53(20.4)$ & $44(15.3)$ & $115(17.7)$ & $91(17.2)$ & $303(17.6)$ & \\
\hline & Unknown* & $18(6.9)$ & $30(10.4)$ & $58(8.9)$ & $41(7.8)$ & $147(8.5)$ & \\
\hline \multirow[t]{3}{*}{ Patient origin } & Born in Spain & $205(78.8)$ & $209(72.6)$ & $478(73.7)$ & $388(73.4)$ & $1280(74.2)$ & \multirow[t]{3}{*}{0.119} \\
\hline & Born outside Spain $^{a}$ & $46(17.7)$ & $73(25.3)$ & $157(24.2)$ & $134(25.3)$ & $410(23.8)$ & \\
\hline & Unknown* & $9(3.5)$ & $6(2.1)$ & $14(2.1)$ & $7(1.3)$ & $36(2.1)$ & \\
\hline \multirow[t]{3}{*}{ Family origin } & Native & $103(39.6)$ & $108(37.5)$ & $0(0.0)$ & $310(58.6)$ & $521(30.2)$ & \multirow[t]{3}{*}{$<0.001$} \\
\hline & Non-native ${ }^{\mathrm{b}}$ & $139(53.5)$ & $169(58.7)$ & $633(97.5)$ & $209(39.5)$ & $1150(66.6)$ & \\
\hline & Unknown* & $18(6.9)$ & $11(3.8)$ & $16(2.5)$ & $10(1.9)$ & $55(3.2)$ & \\
\hline \multirow[t]{3}{*}{ Final diagnosis } & TB-uninfected & $95(36.5)$ & $166(57.6)$ & $557(85.8)$ & $524(99.0)$ & $1342(77.8)$ & \multirow[t]{3}{*}{$<0.001$} \\
\hline & LTBI & $84(32.3)$ & $25(8.7)^{\mathrm{d}}$ & $81(12.5)$ & $5(1.0)$ & $195(11.3)$ & \\
\hline & TBD & $81(31.2)$ & $97(33.7)$ & $11(1.7)$ & $0(0.0)$ & $189(11.0)$ & \\
\hline
\end{tabular}

Abbreviations: $\mathrm{BCG}=$ Bacillus Calmette-Guérin; LTBI = latent tuberculosis infection; TBD = tuberculosis disease; TST = tuberculin skin test.

*Not included in the statistical analysis.

aPatients' origins were: South America (104/410, 25.4\%), Northern Africa (76/410, 18.5\%), Southern Asia (60/410, 14.6\%), Eastern Europe (56/410, 13.7\%),

South-Saharan Africa (53/410, 12.9\%), Far East (26/410, 6.3\%), Western Europe (21/410, 5.1\%), Middle East (13/410, 3.2\%) and North America (1/410, 0.2\%).

'Families' origins were: Northern Africa (422/1150, 36.7\%), South America (266/1150, 23.1\%), Southern Asia (173/1150, 15.0\%), South-Saharan Africa

(100/1150, 8.7\%), Eastern Europe (91/1150, 7.9\%), Far East (49/1150, 4.3\%), Western Europe (34/1150, 3.0\%), Middle East (14/1 150, 1.2\%) and North America $(1 / 1150,0.1 \%)$.

${ }^{c}$ Most common diseases requiring immunosuppressive treatment comprised: malignancies $[\mathrm{n}=225,42.5 \%$; most common diagnoses: neuroblastoma ( $\mathrm{n}=53$ ),

leukaemia $(n=51)$, central nervous system tumor $(n=40)$ and lymphoma $(n=28)]$, rheumatologic diseases $[n=129,24.4 \%$; mainly juvenile idiopathic arthritis

$(\mathrm{n}=95)]$, inflammatory bowel disease $(\mathrm{n}=52,9.8 \%)$, dermatological diseases $[\mathrm{n}=51,9.6 \%$; mainly atopic dermatitis $(\mathrm{n}=23)$ and psoriasis $(\mathrm{n}=18)]$, and COVID-19 $(\mathrm{n}=13,2.5 \%)$.

${ }^{\mathrm{d} P a t i e n t s}$ with incidental diagnosis of LTBI comprised: community-acquired pneumonia $(\mathrm{n}=6)$, self-limiting fever $(\mathrm{n}=4)$, self-limiting cough $(\mathrm{n}=3$ ), self-limiting

hemoptysis $(n=2)$, reactive lymphadenopathy $(n=2)$, and non-tuberculous mycobacterial lymphadenitis, synovitis, Hodgkin's lymphoma, sickle-cell anemia,

bacterial otitis externa, traumatic bone fracture, autoimmune uveitis and self-limiting sacroiliitis ( $\mathrm{n}=1$, each). 


\section{Assay performance in patients with TBD and LTBI}

The overall sensitivity of QFT-Plus in children with TBD was 83.6\% (95\%CI: 77.5-88.6). In subgroup analyses (Table 2), QFT-Plus sensitivity was significantly higher in TST-positive [90.4\% (84.4-94.7)] than in TSTnegative TBD cases [40.7\% (22.4-61.2); $p<0.001]$. Furthermore, the assay sensitivity was higher in patients with microbiologically-confirmed disease [90.1\% (81.5-95.6)] than in cases with unconfirmed disease $[78.7 \%$ (69.886.0); $p=0.036$ ]. No significant differences in sensitivity were observed between patient subgroups based on disease severity and disease location. Compared with TB-uninfected patients, TBD cases were younger, more often born abroad and more commonly born to immigrant families. The latter also had significantly higher ESR values and higher rates of lymphocytosis (Table 3).

The overall sensitivity of QFT-Plus in children with LTBI was 68.2\% (61.2-74.7) (Supplementary Table 2). The sensitivity was significantly higher in children born in Spain [76.0\% (64.8-85.1)] than in those born outside Spain [57.0\% (46.7-66.9); $p=0.009]$, and higher in BCG-unvaccinated children [84.2\% (72.1-92.5)] than in BCG-vaccinated children [46.0\% (35.2-57.0); $p<0.001]$. Compared to TB-uninfected children, patients with LTBI were older, more commonly male, and more commonly BCG-vaccinated (Table 3). In addition, the latter were more commonly born abroad and born to a non-native family.

\section{Correlation between QFT-Plus and tuberculin skin test results}

TSTs were performed in 1188 (68.8\%) patients, of whom 296 (24.9\%) had a positive result. After excluding indeterminate QFT-Plus results, the agreement between QFT-Plus and TST was good in the entire cohort [91.6\%; $\kappa(\mathrm{SE})=0.766(0.022)]$, but only moderate in patients with TBD [86.0\%; $\kappa(\mathrm{SE})=0.472(0.092)]$. TST and QFT-Plus results were concordant in only 88 (54.7\%) of 161 LTBI patients, while 61 (37.9\%) had TST+/QFTPlus- discordance and 12 (7.5\%) had TST-/QFT-Plus+ discordance.

In patients diagnosed with TBD, the sensitivity of TST was $84.4 \%$ (78.1-89.5), very similar to the sensitivity observed with QFT-Plus (83.6\%; $p=0.837$ ). In patients with LTBI, the sensitivity of TST was $92.6 \%$ (87.4-96.1), significantly higher than that of QFT-Plus $(68.2 \% ; p<0.001)$.

\section{QFT-Plus antigen and mitogen responses}

Categorical results (positive/negative) of individual QFT-Plus TB1 and TB2 antigen tubes results were available for 1666 of 1672 (99.6\%) patients with a determinate QFT-Plus result (54 patients with indeterminate results excluded). Overall, the agreement of categorical results between TB1 and TB2 tubes was excellent [98.9\%; $\kappa(\mathrm{SE})=0.961(0.009)]$. Among children with a positive QFT-Plus result, categorical TB1 and TB2 results were concordant in $267(93.7 \%)$ of 284 patients. Eighteen (6.3\%) children showed discordant results: 8 had a TB1+/TB2- and 10 a TB1-/TB2+ constellation. Only 5 of those 18 patients had TBD (TB1+/TB2-, n=2; TB1$/ \mathrm{TB} 2+, \mathrm{n}=3$ ) (Supplementary Table 3). Sex, age, reason for assessment, BCG status and final diagnosis did not differ significantly between those two discordant subgroups (data not shown). 
Table 2. Subgroup analysis of the sensitivity of QFT-Plus in patients with TB disease based on demographic variables, test results and disease

manifestation. Indeterminate QFT-Plus results were considered negative for this analysis. Data are shown as number (percentage), except where stated otherwise.

\begin{tabular}{|c|c|c|c|c|c|c|c|}
\hline & & Total & Positive & Negative & Indeterminate & Sensitivity $(95 \%$ CI $)$ & $p$-value \\
\hline \multicolumn{2}{|l|}{ n (\%) } & $189(100)$ & $158(83.6)$ & $29(15.3)$ & $2(1.1)$ & $83.6(77.5-88.6)$ & \\
\hline \multirow[t]{2}{*}{ Age } & $<5$ years & $89(47.1)$ & $72(80.9)$ & $16(18.0)$ & $1(1.1)$ & $80.9(71.2-88.5)$ & \multirow[t]{2}{*}{0.344} \\
\hline & $\geq 5$ years & $100(52.9)$ & $86(86.0)$ & $13(13.0)$ & $1(1.0)$ & $86.0(77.6-92.1)$ & \\
\hline \multirow[t]{2}{*}{ Sex } & Male & $94(49.7)$ & $76(80.9)$ & $17(18.1)$ & $1(1.1)$ & $80.9(71.4-88.2)$ & \multirow[t]{2}{*}{0.310} \\
\hline & Female & $95(50.3)$ & $82(86.3)$ & $12(12.6)$ & $1(1.1)$ & $86.3(77.7-92.5)$ & \\
\hline \multirow[t]{3}{*}{ BCG status } & Unvaccinated & $139(73.5)$ & $115(82.7)$ & $23(16.5)$ & $1(0.7)$ & $82.7(75.4-88.6)$ & \multirow[t]{3}{*}{0.596} \\
\hline & Vaccinated & $33(17.5)$ & $26(78.8)$ & $6(18.2)$ & $1(3.0)$ & $78.8(61.1-91.0)$ & \\
\hline & Unknown* & $17(9.0)$ & $17(100)$ & $0(0.0)$ & $0(0.0)$ & $100(80.5-100)$ & \\
\hline \multirow[t]{3}{*}{ Patient origin } & Born in Spain & $138(73.0)$ & $115(83.3)$ & $21(15.2)$ & $2(1.5)$ & $83.3(76.1-89.1)$ & \multirow[t]{3}{*}{0.913} \\
\hline & Born outside Spain & $50(26.5)$ & $42(84.0)$ & $8(16.0)$ & $0(0.0)$ & $84.0(70.9-92.8)$ & \\
\hline & Unknown* & $1(0.5)$ & $1(100)$ & $0(0.0)$ & $0(0.0)$ & $100(2.5-100)$ & \\
\hline \multirow[t]{3}{*}{ Family origin } & Native & $43(22.8)$ & $39(90.7)$ & $4(9.3)$ & $0(0.0)$ & 90.7 (77.9-97.4) & \multirow[t]{3}{*}{0.137} \\
\hline & Non-native & $137(72.5)$ & $111(81.0)$ & $24(17.5)$ & $2(1.5)$ & $81.0(73.4-87.2)$ & \\
\hline & Unknown* & $9(4.8)$ & $8(88.9)$ & $1(11.1)$ & $0(0.0)$ & $88.9(51.8-99.7)$ & \\
\hline \multirow[t]{3}{*}{ TST result } & Negative & $27(14.3)$ & $11(40.7)$ & $15(55.6)$ & $1(3.7)$ & $40.7(22.4-61.2)$ & \multirow[t]{3}{*}{$<0.001$} \\
\hline & Positive & $146(77.2)$ & $132(90.4)$ & $13(8.9)$ & $1(0.7)$ & $90.4(84.4-94.7)$ & \\
\hline & Not done* & $16(8.5)$ & $15(93.8)$ & $1(6.3)$ & $0(0.0)$ & $93.8(69.8-99.8)$ & \\
\hline \multirow[t]{3}{*}{ Test indication } & Contact tracing & $81(42.9)$ & $64(79.0)$ & $16(19.8)$ & $1(1.2)$ & $79.0(68.5-87.3)$ & \multirow[t]{3}{*}{0.315} \\
\hline & Clinical / radiological suspicion & $97(51.3)$ & $84(86.6)$ & $13(13.4)$ & $0(0.0)$ & $86.6(78.2-92.7)$ & \\
\hline & New-entrant migrant screening & $11(5.8)$ & $10(90.9)$ & $0(0.0)$ & $1(9.1)$ & $90.9(58.7-99.8)$ & \\
\hline \multirow[t]{2}{*}{ Disease location } & Intrathoracic only & $147(77.8)$ & $121(82.3)$ & $24(16.3)$ & $2(1.4)$ & $82.3(75.2-88.1)$ & \multirow[t]{2}{*}{0.372} \\
\hline & Extrathoracic $+/$ - intrathoracic & $42(22.2)$ & $37(88.1)$ & $5(11.9)$ & $0(0.0)$ & $88.1(74.4-96.0)$ & \\
\hline \multirow[t]{3}{*}{ Disease severity } & Non-severe & $128(67.7)$ & $103(80.5)$ & $23(18.0)$ & $2(1.6)$ & $80.5(72.5-86.9)$ & \multirow[t]{3}{*}{0.130} \\
\hline & Severe & $57(30.2)$ & $51(89.5)$ & $6(10.5)$ & $0(0.0)$ & $89.5(78.5-96.0)$ & \\
\hline & Unknown* & $4(2.1)$ & $4(100)$ & $0(0.0)$ & $0(0.0)$ & $100(39.8-100)$ & \\
\hline \multirow{2}{*}{$\begin{array}{l}\text { Microbiological } \\
\text { confirmation }\end{array}$} & No & $108(57.1)$ & $85(78.7)$ & $21(19.4)$ & $2(1.9)$ & $78.7(69.8-86.0)$ & \multirow[t]{2}{*}{0.036} \\
\hline & Yes & $81(42.9)$ & $73(90.1)$ & $8(9.9)$ & $0(0.0)$ & $90.1(81.5-95.6)$ & \\
\hline
\end{tabular}

Abbreviations: $\mathrm{BCG}=$ Bacillus Calmette-Guérin; $\mathrm{CI}$ = confidence interval; TST = tuberculin skin test. *Not included in the statistical analysis 
Table 3. Comparison of demographic characteristics and test results between TB-uninfected children, patients with latent TB infection (LTBI) and TB disease (TBD). Data are shown as number (percentage) or median (IQR).

\begin{tabular}{|c|c|c|c|c|c|c|}
\hline (2) & 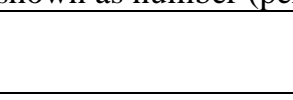 & $\begin{array}{c}\text { TB-uninfected, } \\
\text { n=1342 (77.8) }\end{array}$ & $\begin{array}{c}\text { LTBI } \\
n=195(11.3)\end{array}$ & $\begin{array}{c}\text { TBD } \\
n=189(11.0)\end{array}$ & $\begin{array}{c}\text { TBD vs. TB- } \\
\text { uninfected }\end{array}$ & $\begin{array}{c}\text { LTBI vs. TB- } \\
\text { uninfected }\end{array}$ \\
\hline Age & $<5$ years & $403(30.0)$ & $23(11.8)$ & $89(47.1)$ & $<0.001$ & $<0.001$ \\
\hline & $\geq 5$ years & $939(70.0)$ & $172(88.2)$ & $100(52.9)$ & & \\
\hline Sex & Male & $687(51.2)$ & $130(66.7)$ & $94(49.7)$ & 0.708 & $<0.001$ \\
\hline & Female & $655(48.8)$ & $65(33.3)$ & $95(50.3)$ & & \\
\hline BCG status & Unvaccinated & $1080(80.5)$ & $57(29.2)$ & $139(73.5)$ & 0.106 & $<0.001$ \\
\hline & Vaccinated & $183(13.6)$ & $87(44.6)$ & $33(17.5)$ & & \\
\hline & Unknown* & $79(5.9)$ & $51(26.2)$ & $17(9.0)$ & & \\
\hline Patient origin & Born in Spain & $1067(79.5)$ & $75(38.5)$ & $138(73.0)$ & 0.026 & $<0.001$ \\
\hline & Born outside Spain & $260(19.4)$ & $100(51.3)$ & $50(26.5)$ & & \\
\hline & Unknown* & $15(1.1)$ & $20(10.3)$ & $1(0.5)$ & & \\
\hline Family origin & Native & $438(32.6)$ & $40(20.5)$ & $43(22.8)$ & 0.012 & 0.011 \\
\hline & Non-native & $883(65.8)$ & $130(66.7)$ & $137(72.5)$ & & \\
\hline & Unknown* & $21(1.6)$ & $25(12.8)$ & $9(4.8)$ & & \\
\hline QFT-Plus result & Negative & $1291(96.2)$ & $61(31.3)$ & $29(15.3)$ & $<0.001$ & $<0.001$ \\
\hline & Positive & $0(0.0)$ & $133(68.2)$ & $158(83.6)$ & & \\
\hline & Indeterminate & $51(3.8)$ & $1(0.5)$ & $2(1.1)$ & & \\
\hline TST result $* *$ & Negative & $853(100)$ & $12(7.4)$ & $27(15.6)$ & $<0.001$ & $<0.001$ \\
\hline & Positive & $0(0.0)$ & $150(92.6)$ & $146(84.4)$ & & \\
\hline Haemoglobin level & Low & $219(31.7)$ & $20(13.8)$ & $44(24.4)$ & 0.112 & $<0.001$ \\
\hline & Normal & $441(63.9)$ & $116(80.0)$ & $130(72.2)$ & & \\
\hline & High & $30(4.3)$ & $9(6.2)$ & $6(3.3)$ & & \\
\hline Lymphocyte count & Low & $103(15.0)$ & $4(2.8)$ & $6(3.4)$ & $<0.001$ & $<0.001$ \\
\hline & Normal & $502(73.1)$ & $126(86.9)$ & $137(76.5)$ & & \\
\hline & High & $82(11.9)$ & $15(10.3)$ & $36(20.1)$ & & \\
\hline C-reactive protein & & $2.4(0.5-23.5)$ & $0.7(0.3-3.7)$ & $4.7(1.5-37.9)$ & 0.775 & $<0.001$ \\
\hline Erythrocyte sedime & $\mathrm{n}$ rate $(\mathrm{mm})$ & $4.0(2.0-14.0)$ & $5.0(2.0-13.0)$ & $16.0(5.0-39.5)$ & $<0.001$ & 0.748 \\
\hline
\end{tabular}

Erythrocyte sedimentation rate $(\mathrm{mm})$ $4.0(2.0-14.0)$ $5.0(2.0-13.0)$ $16.0(5.0-39.5)$

*Not included in the statistical analysis.

** TST was only performed in 853 TB-uninfected, 162 LTBI and 173 TBD patients. 
Quantitative data for all four assay tubes were available in 999 patients (57.9\%), and TB1-nil and TB2-nil results in 1367 (79.2\%). In patients with positive QFT-Plus results and available quantitative data ( $\mathrm{n}=284)$, median (IQR) background-corrected TB1 responses were 4.62 IU/ml (2.33-7.13) in TBD cases and 5.17 IU/ml (1.677.38) in LTBI cases ( $p=0.741)$; median TB2 responses were $4.68 \mathrm{IU} / \mathrm{ml}$ (2.26-7.39) and 5.05 IU/ml (1.83-7.33), respectively $(p=0.612)$. There was a strong positive correlation between background-corrected TB1 and TB2 responses $(\mathrm{r}=0.931, p=0.001$; Figure $2 \mathrm{~A}$ ). There was also a weak association between background-corrected TB1 and TB2 responses and TST induration diameters (Figures $2 B \& 2 C$ ), but no association between antigen responses and CRP or ESR (data not shown).

Background-corrected mitogen (positive control) responses showed a statistically significant positive association with age and a significant inverse relationship with CRP concentrations and ESR (Supplementary Figure 1A1C). Haemoglobin or lymphocyte levels had no impact on quantitative QFT-Plus results, neither on antigen nor on mitogen responses (data not shown).

\section{Indeterminate QFT-Plus results}

Overall, 54 patients $(3.1 \%$ ) had an indeterminate QFT-Plus result. In 50 patients the indeterminate result was due to insufficient mitogen responses, while only one patient had that result due to an elevated nil tube interferongamma concentration (quantitative data not available in $n=3$ ). The group of patients with indeterminate results mainly comprised children classified as TB-uninfected $(n=51)$; one child had LTBI, and two had TBD (both unconfirmed).

The bivariate analyses showed that patients with an indeterminate result were on average younger than those with determinate test results (Table 4). Additionally, the analyses showed that the proportion of indeterminate results was highest in children in the CRS and IIT groups. Furthermore, indeterminate results were associated with low haemoglobin concentrations and lymphopenia, as well as elevated CRP and ESR levels.

In the logistic regression model, in which all variables with a $p$-value $<0.1$ in the bivariate analysis were included, age <5 years, lymphopenia, and elevated CRP concentrations remained significantly associated with indeterminate QFT-Plus results (Table 4). 
Table 4. Bivariate statistical analysis and multivariate regression analysis of potential risk factors associated with indeterminate QFT-Plus results.

\begin{tabular}{|c|c|c|c|c|c|c|}
\hline \multirow{2}{*}{ 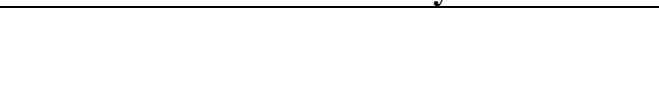 } & \multicolumn{3}{|c|}{ Bivariate analysis } & \multicolumn{3}{|c|}{ Multivariate regression analysis } \\
\hline & Odds ratio & $95 \% \mathrm{CI}$ & $p$-value & Adjusted odds ratio & $95 \% \mathrm{CI}$ & $p$-value \\
\hline Age $<5$ years & 1.924 & 1.11-3.33 & 0.019 & 3.392 & 1.26-9.17 & 0.016 \\
\hline Male sex & 1.674 & $0.95-2.95$ & 0.075 & 3.101 & 1.07-8.96 & $\mathbf{0 . 0 3 7}$ \\
\hline BCG-vaccinated & 1.112 & $0.54-2.31$ & 0.776 & - & - & - \\
\hline Patient born in Spain & 1.125 & $0.59-2.16$ & 0.723 & - & - & - \\
\hline Spanish family origin & 1.646 & $0.94-2.88$ & 0.081 & 2.117 & $0.74-6.03$ & 0.160 \\
\hline Reason for assessment & - & - & - & - & - & - \\
\hline New-entrant migrant screening & Ref. & - & - & Ref. & - & - \\
\hline Clinical/radiological suspicion of TBD & 9.097 & $3.36-24.62$ & $<0.001$ & 0.114 & $0.01-1.62$ & 0.114 \\
\hline Contact tracing & 0.998 & $0.192-5.18$ & 0.999 & - & - & - \\
\hline Prior to immunosuppressive treatment & 7.198 & 2.76-18.78 & $<0.001$ & 0.268 & $0.03-2.81$ & 0.272 \\
\hline Low haemoglobin level ${ }^{\#}$ & 5.855 & 3.05-11.26 & $<0.001$ & 1.137 & $0.39-3.31$ & 0.814 \\
\hline Low lymphocyte count ${ }^{\#}$ & 6.184 & $3.22-11.86$ & $<0.001$ & 4.881 & $1.64-14.50$ & 0.004 \\
\hline CRP level (per 5 mg/L increase) & 1.065 & 1.05-1.09 & $<0.001$ & 1.067 & 1.03-1.10 & $<0.001$ \\
\hline ESR level (per $1 \mathrm{~mm}$ increase) & 1.025 & 1.01-1.04 & $<0.001$ & 1.013 & $0.99-1.03$ & 0.196 \\
\hline
\end{tabular}

Abbreviations: $\mathrm{BCG}=$ Bacillus Calmette-Guérin $\mathrm{CI}=$ confidence interval; $\mathrm{CRP}=\mathrm{C}$-reactive protein; $\mathrm{ESR}=$ erythrocyte sedimentation rate; $\mathrm{TBD}=$ tuberculosis disease .

\#Haemoglobin concentrations and lymphocyte counts were dichotomized (low values $v s$. normal/high values) for this analysis. 


\section{DISCUSSION}

To our knowledge, this is the largest study to date to evaluate QFT-Plus assays in children and adolescents at risk of TB, facilitated by a well-established collaborative paediatric TB research network. Our study was conducted in a real-life clinical setting and included patients assessed for TB infection for the four most common screening indications in low-burden countries.

Our cohort includes a substantial number of children with TBD. TBD was more common in young children, in patients assessed due to recent TB contact or clinical/radiological suspicion, and in those born abroad or to an immigrant family, as expected.[31] In TBD the sensitivity of QFT-Plus was only 83.6\%, equivalent to that of TST $(84.4 \%)$ in our study. Importantly, this figure is also very similar to the estimates reported for previous generation IGRAs in children, including QFT-GIT 83\% (95\%CI: 75-92) and T-SPOT.TB 84\% (95\%CI: 63-10) assays,[7] indicating that the new assay design has not resulted in enhanced sensitivity in TBD patients, as has been claimed.[26] This aligns with data from adult studies that performed head-to-head comparisons of QFTGIT and QFT-Plus in adult TBD patients, reporting nearly identical sensitivities for both assays.[13] While it would have been desirable to include a direct head-to-head comparison with previous generation IGRAs in our study, few paediatric IGRA studies include such comparisons due to the technical difficulties in obtaining large enough blood samples from young children. One paediatric study in Eswatini, which included only 12 children with TBD, directly compared QFT-GIT and QFT-Plus, and reported identical sensitivities.[17]

To date, the data on the performance of QFT-Plus in children with TBD remain very limited, despite the assay having been released for routine clinical use in 2016. The two largest published paediatric studies in Europe, which included 43 children with suspected TBD and 84 children with microbiologically-confirmed TBD, reported test sensitivities of $83.3 \%$ and $82.9 \%$, respectively.[19,20] Concerningly, one paediatric study in Vietnam, a high TB prevalence setting, reported that while the sensitivity of QFT-Plus was $84.2 \%$ in patients with isolated pulmonary TBD $(n=19)$, it was only $14.3 \%$ in patients with extrapulmonary TBD $(n=14) .[18]$

Subgroup analyses showed that the sensitivity of QFT-Plus was significantly higher in microbiologicallyconfirmed than in unconfirmed TBD cases. Although the underlying mechanism is uncertain, it is tempting to hypothesise that this may have resulted from the former patient group having more advanced disease and higher antigenic loads, leading to greater induction of MTB-specific T cells, and thereby higher interferon-gamma responses in the QFT-Plus assay. This hypothesis aligns with previous adult data indicating that there is a relationship between antigenic load and the magnitude of interferon-gamma responses.[32] Nevertheless, it is possible that some children in our study who lacked microbiological confirmation had an alternative diagnosis and were incorrectly diagnosed with TBD. We also found that the sensitivity of QFT-Plus was significantly higher in TST-positive than in TST-negative TBD cases, which is not unexpected, as both tests are based on the detection of immune responses to mycobacterial antigens.

According to the manufacturer, the second QFT-Plus antigen tube (TB2), which was absent from previous assay versions, is intended to preferentially induce MTB-specific CD8+ T cell responses to improve the assay's 
sensitivity specifically in patients with TBD (rather than LTBI).[26] Only 5 (2.6\%) of 189 children with TBD in our cohort had discordance between categorical TB1 and TB2 results, and only 3 (1.6\%) of those had a TB1/TB2+ result constellation, showing that the contribution of the second antigen tube to the assay's sensitivity in paediatric TBD is negligible. Importantly, the disadvantages of having an additional antigen tube include the need to obtain a larger volume of blood, which can be challenging in young children, and a substantial increase in costs due to the need to run an additional sample for every patient in the QFT ELISA. In contrast to the TST, all IGRAs require a complex laboratory infrastructure, which is a challenge in low-resource, high TB incidence countries. Notably, recent data suggest that skin tests based on ESAT-6 and CFP-10, which are likely to have greater specificity than the TST that uses a mixture of poorly-defined mycobacterial antigens, may be a viable alternative in settings with limited infrastructure.[33]

The analyses of quantitative antigen-induced interferon-gamma responses revealed a strong positive correlation between TB1 and TB2 responses, and only a weak relationship between antigen-induced interferon-gamma responses and TST indurations. Importantly, the magnitude of TB1 and TB2 responses did not differ significantly between LTBI and TBD cases, showing that the assay is not suited to differentiating between those infection states. We observed a positive correlation between age and the magnitude of mitogen responses, a phenomenon that has also been reported in QFT-GIT.[34] Furthermore, we found that there was an inverse relationship between mitogen responses and CRP concentrations and ESRs, indicating that pro-inflammatory states can have an adverse impact on positive control responses.[35]

LTBI was diagnosed in 195 patients in our cohort, most often among children assessed during CTR and NMS. The sensitivity of QFT-Plus for LTBI was $68.2 \%$ overall, and was significantly higher in BCG-unvaccinated children and those born in Spain, likely reflecting result discordance between TST and QFT-Plus in BCGvaccinated patients. Importantly, comparisons between studies investigating the performance of diagnostic tests for LTBI are hampered by the lack of a gold standard and the presence of varying TB risk factors in the populations assessed.[36] However, we recently reported a similar sensitivity estimate (72.2\%) for the QFT-GIT assay in the diagnosis of LTBI among children $<5$ years-of-age.[37]

The proportion of indeterminate assay results (3.1\%) was very similar to that reported by a recent meta-analysis on previous-generation IGRAs in children (4\%),[38] and those results were almost universally due to low mitogen (positive-control) responses. We found that indeterminate QFT-Plus results were associated with young age, a phenomenon previously also reported in QFT-GIT assays.[34,37,39] Multivariate analysis additionally identified lymphopenia and elevated CRP as significant risk factors for indeterminate QFT-Plus results. The former is to be expected, as IGRAs require a sufficient number of functional $\mathrm{T}$ cells in order to generate adequate interferon-gamma responses, and - in contrast to the T-SPOT.TB assay - cell numbers are not being adjusted prior to antigenic stimulation in QFT assays. Interestingly, recent data suggest that CRP at physiological concentrations can inhibit intracellular expression of various cytokines, including IL-2 and interferon-gamma, which may explain the latter observation.[35] 
One of the key strengths of this study lies in its sample size, allowing us to generate precise sensitivity estimates with narrow confidence intervals. Another strength is that the study was conducted in a routine clinical setting, as diagnostic studies conducted under highly-controlled conditions tend to overestimate test performance. However, our study has limitations, including the absence of direct head-to-head comparisons with other IGRA assays and missing data (including TST results, haematological results and inflammatory markers) in a substantial number of patients. Also, we did not collect data on the precise timing of the TST and blood sampling for QFT-Plus; while data from early studies suggested that performing a TST prior to an IGRA may result in a boosting phenomenon, this has subsequently been shown not to be the case.[40-42] In common with all previous studies, our estimates of test performance related to the diagnosis of LTBI are limited by the lack of a universally-agreed gold standard, and the definition of LTBI used may have led to incorporation bias.

In summary, our results show that in children and adolescents at risk of TB in a low TB prevalence setting the QFT-Plus assay does not perform better than the previous-generation IGRAs or the long-established TST, consistent with the data of the largest meta-analysis in adults to date.[43] Close to one in five children with TBD had false-negative QFT-Plus results, highlighting that the assay can not be used as a rule-out test in children with suspected TBD. Furthermore, our data show that the new antigen tube, TB2, only makes a negligible contribution to assay sensitivity. Multivariate analysis identified young age, lymphopenia and elevated CRP concentrations as risk factors for indeterminate test result. Further studies are needed to determine whether QFTPlus assays perform better than previous generation IGRAs in children in high TB prevalence settings. 


\section{Funding and conflicts of interest}

This work was partially supported by a research grant from the Carlos III Institute of Health, Ministry of Economy and Competitiveness (Spain), reference PI16/00314, and by the Spanish Society of Pneumology and Thoracic Surgery, grant number 90/2015. Tomàs PÉREZ-PORCUNA and Antoni NOGUERA-JULIAN were supported by "Subvencions per a la Intensificació de Facultatius Especialistes" (Departament de Salut de la Generalitat de Catalunya, Programa PERIS 2016-2020) [SLT006/17/00144 and SLT008/18/00193, respectively]. Marc TEBRUEGGE has received QuantiFERON assays at reduced pricing or free of charge for TB diagnostics projects from the manufacturer (Cellestis/Qiagen) in the past, and has received support for conference attendance from Cepheid. The manufacturers had no influence on the study design, data collection, analysis or interpretation, writing of the manuscript or decision to submit the data for publication. The remaining authors have no conflicts of interest to disclose. 


\section{REFERENCES}

1. Lawn SD, Zumla AI. Tuberculosis. Lancet 2011;378:57-72.

2. WHO. Global tuberculosis report 2018. ww.who.int/tb/publications/global_report/en/. Date last accessed: April 252021.

3. Nelson LJ, Wells CD. Global epidemiology of childhood tuberculosis. Int J Tuberc Lung Dis 2004;8:636-47.

4. Marais BJ, Gie RP, Schaaf HS, et al. Childhood pulmonary tuberculosis: old wisdom and new challenges. Am J Respir Crit Care Med 2006;173:1078-90.

5. Mandal N, Anand PK, Gautam S, et al. Diagnosis and treatment of paediatric tuberculosis: An insight review. Crit Rev Microbiol 2017;43:466-80.

6. Bonilla FA, Khan DA, Ballas ZK, et al. Practice parameter for the diagnosis and management of primary immunodeficiency. J Allergy Clin Immunol 2015;136:1186-205.

7. Mandalakas AM, Detjen AK, Hesseling AC, et al. Interferon-gamma release assays and childhood tuberculosis: systematic review and meta-analysis. Int $J$ Tuberc Lung Dis 2011;15:1018-32.

8. Tebruegge M, Ritz N, Koetz K, et al. Availability and Use of Molecular Microbiological and Immunological Tests for the Diagnosis of Tuberculosis in Europe. PLoS One 2014;9:e99129.

9. Starke JR. Interferon- $\gamma$ Release Assays for Diagnosis of Tuberculosis Infection and Disease in Children. Pediatrics 2014;134:e1763-e73.

10. Ryu MR, Park MS, Cho EH, et al. Comparative Evaluation of QuantiFERON-TB Gold In-Tube and QuantiFERON-TB Gold Plus in Diagnosis of Latent Tuberculosis Infection in Immunocompromised Patients. J Clin Microbiol 2018;56:e00438-18.

11. Petruccioli E, Chiacchio T, Pepponi I, et al. First characterization of the CD4 and CD8 T-cell responses to QuantiFERON-TB Plus. J Infect 2016;73:588-97.

12. Pourakbari B, Mamishi S, Benvari S, et al. Comparison of the QuantiFERON-TB Gold Plus and QuantiFERON-TB Gold In-Tube interferon- $\gamma$ release assays: A systematic review and metaanalysis. Adv Med Sci 2019;64:437-43.

13. Shafeque A, Bigio J, Hogan CA, et al. Fourth-generation quanti FERON-TB gold plus: What is the evidence? J Clin Microbiol 2020;58:e01950-19.

14. Petruccioli E, Vanini V, Chiacchio T, et al. Analytical evaluation of QuantiFERON- Plus and QuantiFERON- Gold In-tube assays in subjects with or without tuberculosis. Tuberculosis (Edinb) 2017;106:38-43.

15. Petruccioli E, Chiacchio T, Navarra A, et al. Effect of HIV-infection on QuantiFERON-plus accuracy in patients with active tuberculosis and latent infection. $J$ Infect 2020;80:536-46.

16. Chiacchio T, Petruccioli E, Vanini V, et al. Characterization of QuantiFERON-TB-Plus results in latent tuberculosis infected patients with or without immune-mediated inflammatory diseases. J Infect 2019;79:15-23. 
17. Kay AW, DiNardo AR, Dlamini Q, et al. Evaluation of the QuantiFERON-Tuberculosis Gold Plus Assay in Children with Tuberculosis Disease or Following Household Exposure to Tuberculosis. Am J Trop Med Hyg 2019;100:540-3.

18. Nguyen DT, Phan H, Trinh T, et al. Sensitivity and characteristics associated with positive QuantiFERON-TB Gold-Plus assay in children with confirmed tuberculosis. PLoS One 2019;14:e0213304.

19. Buonsenso D, Delogu G, Perricone C, et al. Accuracy of QuantiFERON-TB Gold-PLUS Test for the Diagnosis of Mycobacterium tuberculosis infection in Children. J Clin Microbiol 2020;58:e00272-20.

20. Soler-Garcia A, Gamell A, Santiago B, et al. Diagnostic Accuracy of QuantiFERON-TB Gold Plus Assays in Children and Adolescents with Tuberculosis Disease. J Pediatr 2020;223:212-5.

21. Primaturia C, Reniarti L, Nataprawira HMN. Comparison between the Interferon $\gamma$ Release Assay - QuantiFERON Gold plus (QFT-Plus) - And Tuberculin Skin Test (TST) in the Detection of Tuberculosis Infection in Immunocompromised Children. Pulm Med 2020;2020:7159485.

22. Soler-Garcia A, Gamell A, Santiago B, et al. QuantiFERON-TB Gold Plus assay specificity in children and adolescents with suspected tuberculosis - a multi-center cross-sectional study in Spain. Pediatr Infect Dis J 2021;40:e348-e351.

23. Piñeiro Pérez R, Santiago García B, José Mellado Peña M. Red Española de Estudio de Tuberculosis Pediátrica. A la vanguardia de Europa. Rev Esp Salud Publica 2017;91:e201701001.

24. Harris PA, Taylor R, Thielke R, et al. Research electronic data capture (REDCap)--a metadatadriven methodology and workflow process for providing translational research informatics support. J Biomed Inform 2009;42:377-81.

25. Grupo de trabajo Plan Prevención y Control de la Tuberculosis. Plan para la prevención y control $\begin{array}{lllll}\text { de la } & \text { tuberculosis } & \text { en } & \text { España }\end{array}$ www.mscbs.gob.es/profesionales/saludPublica/prevPromocion/PlanTuberculosis/docs/PlanTB20 19.pdf. Date last accessed: April 252021.

26. QIAGEN. QuantiFERON-TB Gold Plus (QFT-Plus) ELISA Package Insert. Rev. 04. www.QuantiFERON.com. Date last accessed: April 252021.

27. Moreno-Pérez D, Andrés Martín A, Altet Gómez N, et al. Diagnosis of tuberculosis in pediatrics. Consensus document of the Spanish Society of Pediatric Infectology (SEIP) and the Spanish Society of Pediatric Pneumology (SENP). An Pediatr (Barc) 2010;7:143.e1-143.14.

28. Mayo Clinic Laboratories. Complete Blood Count (CBC) with Differential, Blood. www.mayocliniclabs.com/test-info/pediatric/refvalues/reference.php?unit code=9109. Date last accessed: April 252021.

29. Graham SM, Ahmed T, Amanullah F, et al. Evaluation of tuberculosis diagnostics in children: 1. Proposed clinical case definitions for classification of intrathoracic tuberculosis disease. Consensus from an expert panel. J Infect Dis 2012;205:S199-208. 
30. Wiseman CA, Gie RP, Starke JR, et al. A proposed comprehensive classification of tuberculosis disease severity in children. Pediatr Infect Dis $J$ 2012;31:347-52.

31. European Centre for Disease Prevention and Control/WHO Regional Office. Tuberculosis surveillance and monitoring in Europe $2018 \quad-\quad 2016$ data. www.ecdc.europa.eu/sites/portal/files/documents/ecdc-tuberculosis-surveillance-monitoringEurope-2018-19mar2018.pdf 28. Date last accessed: April 252021.

32. Millington KA, Innes JA, Hackforth S, et al. Dynamic relationship between IFN-gamma and IL2 profile of Mycobacterium tuberculosis-specific $\mathrm{T}$ cells and antigen load. $J$ Immunol 2007;178:5217-26.

33. Hamada Y, Cirillo DM, Matteelli A, et al. Tests for tuberculosis infection: landscape analysis. Eur Respir J 2021 Apr 19:2100167. Epub ahead of print.

34. Connell TG, Tebruegge M, Ritz N, et al. Indeterminate interferon-gamma release assay results in children. Pediatr Infect Dis $J$ 2010;29:285-6.

35. Yoshida T, Ichikawa J, Giuroiu I, et al. C reactive protein impairs adaptive immunity in immune cells of patients with melanoma. J Immunother Cancer 2020;8:e000234.

36. Auguste P, Tsertsvadze A, Pink J, et al. Comparing interferon-gamma release assays with tuberculin skin test for identifying latent tuberculosis infection that progresses to active tuberculosis: Systematic review and meta-analysis. BMC Infect Dis 2017;17:200.

37. Velasco-Arnaiz E, Soriano-Arandes A, Latorre I, et al. Performance of Tuberculin Skin Tests and Interferon- $\gamma$ Release Assays in Children Younger Than 5 Years. Pediatr Infect Dis $J$ 2018;37:1235-41.

38. Meier NR, Volken T, Geiger M, et al. Risk factors for indeterminate interferon-gamma release assay for the diagnosis of tuberculosis in children - A systematic review and meta-analysis. Front Pediatr 2019;7:208.

39. Tebruegge M, de Graaf H, Sukhtankar P, et al. Extremes of age are associated with indeterminate QuantiFERON-TB gold assay results. J Clin Microbiol 2014;52:2694-7.

40. Richeldi L, Bergamini BM, Vaienti F. Prior tuberculin skin testing does not boost QuantiFERON-TB results in paediatric contacts. Eur Respir J 2008;32:524-5.

41. Ritz N, Yau C, Connell TG, et al. Absence of interferon-gamma release assay conversion following tuberculin skin testing. Int J Tuberc Lung Dis 2011;15:767-9.

42. Velasco-Arnaiz E, Soriano-Arandes A, Espiau M, et al. Impact of Baseline Tuberculin Skin Test and Isoniazid Chemoprophylaxis on Subsequent Quantiferon-TB Gold In-Tube Performance in Young Children Assessed After Tuberculosis Contact in Catalonia. Pediatr Infect Dis J 2020;39:e22-5.

43. Oh CE, Ortiz-Brizuela E, Bastos ML, et al. Comparing the Diagnostic Performance of QuantiFERON-TB Gold Plus to Other Tests of Latent Tuberculosis Infection: A Systematic Review and Meta-analysis. Clin Infect Dis 2021;73:e1116-25. 


\section{Figures legends}

Figure 1. Flow-chart showing the indications for TB screening, as well as QFT-Plus assay results and the final diagnoses regarding TB. Abbreviations: LTBI = latent tuberculosis infection; QFT-Plus = QuantiFERON-TB Gold Plus; TB = tuberculosis; TBD = tuberculosis disease.

Figure 2. Relationship between (A) background-corrected interferon-gamma concentrations in TB1 antigenstimulated samples (TB1-nil) and TB2 antigen-stimulated samples (TB2-nil) in 284 patients with a positive QFT-Plus result in whom quantitative data were available; (B) tuberculin skin test diameter and backgroundcorrected interferon-gamma concentrations in TB1 antigen-stimulated samples (TB1-nil); and (C) tuberculin skin test diameter and background-corrected interferon-gamma concentrations in TB2 antigen-stimulated sample (TB2-nil). All graphs show a fitted linear regression line (solid line) and the corresponding 95\% confidence interval (interrupted lines). The values shown are Spearman correlation coefficients $(r)$ and their corresponding $p$-value. 